\title{
Semiclassical description of Heisenberg models via spin-coherent states
}

\author{
John Schliemann and Franz G. Mertens \\ Physikalisches Institut, Universität Bayreuth, D-95440 Bayreuth, Germany
}

November 1997

\begin{abstract}
We use spin-coherent states as a time-dependent variational ansatz for a semiclassical description of a large family of Heisenberg models. In addition to common approaches we also evaluate the square variance $\left\langle\mathcal{H}^{2}\right\rangle-\langle\mathcal{H}\rangle^{2}$ of the Hamiltonian in terms of coherent states. This quantity turns out to have a natural interpretation with respect to timedependent solutions of the equations of motion and allows for an estimate of quantum fluctuations in a semiclassical regime. The general results are applied to solitons, instantons and vortices in several one- and two-dimensional models.

PACS numbers: $75.10 . \mathrm{Hk}, 75.10 . \mathrm{Jm}, 03.65 . \mathrm{Sq}$
\end{abstract}

\section{Introduction}

There has been a lot of study on classical spin systems in the last decades, commonly using a continuum description, for a review see Kosevich et al. 1990. Some one-dimensional models have been identified as integrable and treated by means of the inverse scattering method (Takhtajan 1977, Fogedby 1980, Sklyanin 1979). A common feature of such nonlinear evolution equations is the existence of localized solutions like solitary waves and, in the integrable case, mathematical solitons.

In quantum spin systems analytical treatments appear to be restricted to one-dimensional models. For the spin- $\frac{1}{2}$-Heisenberg chain of $N$ lattice sites Bethe (1931) managed to reduce the diagonalisation of the Hamiltonian acting on a $2^{N}$-dimensional Hilbert space to the problem of solving $N$ coupled nonlinear algebraic equations, which are well understood in the thermodynamic limit. By this Bethe ansatz and its algebraic formulation many properties of the model such as thermodynamic quantities could be extracted. Nevertheless, the eigenstates of the Hamiltonian themselves are obtained only rather implicitly and are naturally translationally symmetric, i .e. non localized. Thus, a quantum analogon to a classical timedependent soliton, which should not be an eigenstate of energy but a superposition of them, has not been found yet.

So it is desirable to give a description of solitons in the semiclassical regime, i.e. for large but finite values of the spin length. Considerable work in this direction has been done using different kinds of bosonization and tensor products of coherent oscillator states as variational ansatz, see e. g. De Azevedo et al. 1982, Ferrer and Pozo 1988, Kapor et al. 1990. Disadvantages of these methods are difficulties in treating infinite series of nontrivial operator products 
and, more fundamental, the problem of mapping the finite dimensional Hilbert space of a spin onto an infinite dimensional bosonic space.

An alternative to such approaches are spin-coherent states as introduced by Radcliffe (1971). Balakrishnan, Bishop $(1985,89)$ and Balakrishnan et al. (1990) used these objects for treating ferromagnetic Heisenberg chains and evaluated quantum corrections to classical soliton dispersion laws. In detail, their way of proceeding has been subject to some criticism by Haldane (1986), but the general findings concerning soliton stability with respect to quantum fluctuation seem to be valid and will be confirmed in the present work. Moreover, Frahm and Holyst (1989) suggested an extension of spin-coherent states introducing a squeezing similar to bosonic states. Unfortunately, this approach leads for generic models to very complicated equations of motion for the classical angular variables and the additional squeeze parameter, that cannot be solved exactly for nontrivial cases.

In this paper we follow the above authors and use (unsqueezed) spin-coherent states as variational ansatz for a large family of Heisenberg models. In addition to the quantities considered in the above references we also calculate the quantum mechanical variance of the energy. This quantity can either be used as a test of the validity of the approach; on the other hand, the general expression obtained has a natural physical interpretation concerning energy fluctuations in time-dependent spin structures. Finally we apply our results to several prominent solitary solutions of spin models.

\section{Coherent states}

In the Hilbert space of a spin of length $S$ we define a spin-coherent state $|S ; \vartheta, \varphi\rangle$ by the equation

$$
\vec{s}_{\vartheta, \varphi} \cdot \hat{\vec{S}}|S ; \vartheta, \varphi\rangle=\hbar S|S ; \vartheta, \varphi\rangle
$$

for the direction $\vec{s}_{\vartheta, \varphi}=(\sin \vartheta \cos \varphi, \sin \vartheta \sin \varphi, \cos \vartheta)$. In the usual basis of eigenstates of $\hat{S}^{z}$ $\left(\hat{S}^{z}|m\rangle=\hbar m|m\rangle\right)$ these states can be expressed as

$$
|S ; \vartheta, \varphi\rangle=U(\vartheta, \varphi)|S\rangle=\sum_{n=0}^{2 S}\left(\begin{array}{c}
2 S \\
n
\end{array}\right)^{\frac{1}{2}}\left(\cos \left(\frac{\vartheta}{2}\right)\right)^{2 S-n}\left(\sin \left(\frac{\vartheta}{2}\right)\right)^{n} e^{\imath \varphi(n-S)}|S-n\rangle
$$

with

$$
U(\vartheta, \varphi)=\exp \left(-\frac{\imath}{\hbar} \varphi \hat{S}^{z}\right) \exp \left(-\frac{\imath}{\hbar} \vartheta \hat{S}^{y}\right)
$$

Clearly, we have $\langle S ; \vartheta, \varphi|\hat{\vec{S}}| S ; \vartheta, \varphi\rangle=\hbar S \vec{s}_{\vartheta, \varphi}$, but in the expectation values of higher products of spin components contributions of different order in the spin length $S$ arise. A list of diagonal elements useful for the following is given appendix A, where we also demonstrate the classical limit of such expressions.

The spin-coherent states have the minimum uncertaincy product

$$
\Delta\left(\vec{e}_{1} \cdot \hat{\vec{S}}\right) \Delta\left(\vec{e}_{2} \cdot \hat{\vec{S}}\right)=\frac{\hbar}{2} \Delta\left(\vec{e}_{3} \cdot \hat{\vec{S}}\right)
$$

with $\Delta(\hat{O})$ being the variance of an operator $\hat{O}$ and $\vec{e}_{1}, \vec{e}_{2}, \vec{e}_{3}$ an orthonormal system. Furthermore, they fullfill the (over-) completeness relation

$$
\frac{2 S+1}{4 \pi} \int_{0}^{2 \pi} d \varphi \int_{0}^{\pi} d \vartheta \sin \vartheta|S ; \vartheta, \varphi\rangle\langle S ; \vartheta, \varphi|=\mathbf{1}
$$


Nevertheless, it should be mentioned that for an arbitrary linear combination of coherent states a direction $\vec{s}_{\vartheta, \varphi}$ solving eq. (11) cannot be found. Therefore the spin-coherent states do not form a subspace of the Hilbert space, but a submanifold diffeomorphic to the two-dimensional unit sphere. Only in the case $S=\frac{1}{2}$ every (normalized) state vector can be identified as a coherent state.

\section{Time-dependent variational method}

Let us consider the following class of one-dimensional spin models

$$
\mathcal{H}=\sum_{n} \sum_{i=1}^{3}\left[\alpha_{i} \hat{S}_{n}^{i} \hat{S}_{n+1}^{i}+\beta_{i} \hat{S}_{n}^{i} \hat{S}_{n}^{i}+\gamma_{i} \hat{S}_{n}^{i}\right]
$$

The index $i$ runs over the three spatial directions $x, y, z$. Each lattice site labelled by $n$ carries a spin of uniform length $S$. The Hamiltonian includes an anisotropic exchange coupling between nearest neighbors, a local anisotropy and a magnetic field. The parameters $\alpha_{i}, \beta_{i}, \gamma_{i}$ may be chosen arbitrarily, in particular with respect to their sign.

We now employ the direct product of coherent states

$$
|\psi(t)\rangle=\bigotimes_{n}\left|S ; \vartheta_{n}, \varphi_{n}\right\rangle
$$

as a time-dependent variational ansatz, i.e. we assume the time evolution of the state $|\psi(t)\rangle$ under the above Hamiltonian to be given in terms of time-dependent functions $\vartheta_{n}(t), \varphi_{n}(t)$. With $\langle\cdot\rangle$ denoting an expectation value within (7), $\vec{s}_{n}=\left(\sin \vartheta_{n} \cos \varphi_{n}, \sin \vartheta_{n} \sin \varphi_{n}, \cos \vartheta_{n}\right)$ and cartesian directions $\vec{e}^{i}$, we have for $\mathcal{H}$

$$
\langle\mathcal{H}\rangle=(\hbar S)^{2} \sum_{n} \sum_{i=1}^{3}\left[\alpha_{i}\left(\vec{s}_{n} \cdot \vec{e}^{i}\right)\left(\vec{s}_{n+1} \cdot \vec{e}^{i}\right)+\left(1-\frac{1}{2 S}\right) \beta_{i}\left(\vec{s}_{n} \cdot \vec{e}^{i}\right)^{2}+\frac{\gamma_{i}}{\hbar S}\left(\vec{s}_{n} \cdot \vec{e}^{i}\right)\right],
$$

and from the Heisenberg equations of motion $\frac{d}{d t} \hat{\vec{S}}_{n}=-\frac{\imath}{\hbar}\left[\hat{\vec{S}}_{n}, \mathcal{H}\right]$ :

$$
\begin{gathered}
\frac{d}{d t} \vec{s}_{n}=(\hbar S) \sum_{i=1}^{3}\left[\alpha_{i}\left(\vec{s}_{n} \times \vec{e}^{i}\right)\left(\left(\vec{s}_{n-1}+\vec{s}_{n+1}\right) \cdot \vec{e}^{i}\right)+2\left(1-\frac{1}{2 S}\right) \beta_{i}\left(\vec{s}_{n} \times \vec{e}^{i}\right)\left(\vec{s}_{n} \cdot \vec{e}^{i}\right)\right. \\
\left.+\frac{\gamma_{i}}{\hbar S}\left(\vec{s}_{n} \times \vec{e}^{i}\right)\right]
\end{gathered}
$$

The quantity $(\hbar S)$ becomes the classical spin length in the limit $S \rightarrow \infty, \hbar \rightarrow 0, \hbar S=$ const. Eqs. (8), (9) are identical to the classical energy and the Landau-Lifshitz-equation up to a renormalisation of the local anisotropy by a factor $\left(1-\frac{1}{2 S}\right)$, reflecting the fact that this term does not contribute to the dynamics for $S=\frac{1}{2}$. Therefore the variational ansatz provides semiclassical corrections to the equation of motion and reproduces the correct classical limit. This property of spin-coherent states has been found by several authors before with respect to particular spin models, see e. g. the references given in the introduction, and is proved in appendix B for an arbitrary Hamiltonian.

Next we examine the square variance of the Hamiltonian, i. e. $\left\langle\mathcal{H}^{2}\right\rangle-\langle\mathcal{H}\rangle^{2}$. This quantity 
is nonzero only in the quantum case and (as well as $\langle\mathcal{H}\rangle$ ) strictly an invariant of the system, whatever the exact quantum mechanical time evolution of the state (7) will be. After extensive algebra one ends up with the following expression:

$$
\begin{aligned}
&\left\langle\mathcal{H}^{2}\right\rangle-\langle\mathcal{H}\rangle^{2}= \\
&(\hbar S)^{4} \sum_{n}\left[\frac { 1 } { 2 S } \left(\sum _ { i = 1 } ^ { 3 } \left[\alpha_{i}\left(\vec{s}_{n} \times \vec{e}^{i}\right)\left(\left(\vec{s}_{n-1}+\vec{s}_{n+1}\right) \cdot \vec{e}^{i}\right)\right.\right.\right.\left.\left.\gamma_{i}\left(\vec{s}_{n} \times \vec{e}^{i}\right)\right]\right)^{2} \\
&++2\left(1-\frac{1}{2 S}\right) \beta_{i}\left(\vec{s}_{n} \times \vec{e}^{i}\right)\left(\vec{s}_{n} \cdot \vec{e}^{i}\right)+\frac{\gamma^{\prime} S}{\hbar S^{2}}\left(\sum _ { i , j = 1 } ^ { 3 } \left[\alpha _ { i } \alpha _ { j } \left(\left(\left(\vec{s}_{n} \times \vec{e}^{i}\right) \cdot\left(\vec{s}_{n} \times \vec{e}^{j}\right)\right)\right.\right.\right. \\
&+\frac{1}{\left(\left(\vec{s}_{n-1} \times \vec{e}^{i}\right) \cdot\left(\vec{s}_{n-1} \times \vec{e}^{j}\right)+\left(\vec{s}_{n+1} \times \vec{e}^{i}\right) \cdot\left(\vec{s}_{n+1} \times \vec{e}^{j}\right)\right)} \\
&\left.\left.\quad+2 \sum_{i, j=1}^{3}\left[\left(1-\frac{1}{2 S}\right) \beta_{i} \beta_{j}\left(\left(\vec{s}_{n} \times \vec{e}^{i}\right)^{2}\left(\vec{s}_{n} \times \vec{e}^{j}\right)^{2}-\left(\vec{s}_{n} \cdot\left(\vec{e}^{i} \times \vec{e}^{j}\right)\right)^{2}\right)\right]\right)\right]
\end{aligned}
$$

The square variance of the energy consists essentially of two contributions being of order $\frac{1}{S}$ and $\frac{1}{S^{2}}$ respectively. In the above summation over the lattice sites the squared expression in the term of order $\frac{1}{S}$ can be recognized as the r.h.s. of eq. (9). Thus, we have

$$
\left\langle\mathcal{H}^{2}\right\rangle-\langle\mathcal{H}\rangle^{2}=(\hbar S)^{2} \sum_{n} \frac{1}{2 S}\left(\frac{d}{d t} \vec{s}_{n}\right)^{2}+O\left(\frac{1}{S^{2}}\right) .
$$

Within our variational approach the leading order of the quantum fluctuations of the energy is purely due to the time-dependence of the state vector. On the other hand, for a quantum state which has a nontrivial time evolution and is consequently not an eigenstate of the Hamiltonian, the energy must definetely have a finite uncertaincy. Following this observation the leading term in (11) is certainly not an artifact of the ansatz (7), but it is a physically relevant expression for the energy fluctuations in time-dependent semiclassical spin structures. So we have found good evidence that the ansatz of spin-coherent states does not only reproduce the classical limit but is still meaningfull for a semiclassical description. The contributions of order $\frac{1}{S^{2}}$ in (10) cannot be interpretated in a general way and should be studied in the context of particular models. We will see that these terms can often be considered as a criterion for the validity of the variational method.

For brevity we have concentrated in (6) on an one-dimensional model. For higher dimensions one simply has to infer the appropriate number of neighbors of each lattice site in the summations. The result can always be written in the form (11). Moreover, it is also straightforeward to see that the result (11) is still valid in the case of exchange couplings of longer range.

Thus, our above findings apply to a large variety of (ferromagnetic or antiferromagnetic) spin models in arbitrary dimension. This will be illustrated in the next section with respect to several one- and two-dimensional ferromagnetic models. 


\section{Application to solitons, instantons and vortices}

We now calculate the quantum mechanical energy uncertaincy for solitary solutions in different Heisenberg models. We denote the leading order in $\frac{1}{S}$ by $\Omega_{1}$ (cf. eq. (11)), the remaining contributions by $\Omega_{2}$, i.e. $\left\langle\mathcal{H}^{2}\right\rangle-\langle\mathcal{H}\rangle^{2}=\Omega_{1}+\Omega_{2}$.

(i) A ferromagnetic Heisenberg chain with isotropic exchange coupling and an external field is given by

$$
\mathcal{H}_{1}=-J \sum_{n}\left[\hat{\vec{S}}_{n} \hat{\vec{S}}_{n+1}+\hat{\vec{S}}_{n} \vec{B}\right]
$$

with $J>0$. Eqs. (8)-(10) read:

$$
\begin{aligned}
&\left\langle\mathcal{H}_{1}\right\rangle=-(\hbar S)^{2} J \sum_{n}\left[\vec{s}_{n} \vec{s}_{n+1}+\vec{s}_{n} \frac{\vec{B}}{\hbar S}\right] \\
& \frac{d}{d t} \vec{s}_{n}=(\hbar S) J\left(\vec{s}_{n} \times\left(\vec{s}_{n-1}+\vec{s}_{n+1}\right)+\vec{s}_{n} \times \frac{\vec{B}}{\hbar S}\right) \\
&\left\langle\mathcal{H}_{1}^{2}\right\rangle-\left\langle\mathcal{H}_{1}\right\rangle^{2}=(\hbar S)^{4} J^{2} \sum_{n}\left[\frac{1}{2 S}\left(\vec{s}_{n} \times\left(\vec{s}_{n-1}+\vec{s}_{n+1}\right)+\vec{s}_{n} \times \frac{\vec{B}}{\hbar S}\right)^{2}\right. \\
&\left.+\frac{1}{8 S^{2}}\left(\left(1-\vec{s}_{n} \vec{s}_{n-1}\right)^{2}+\left(1-\vec{s}_{n} \vec{s}_{n+1}\right)^{2}\right)\right]
\end{aligned}
$$

We choose appropriate units with $J=1, \hbar S=1$ and the lattice spacing $a=1$ and calculate the expectation value of (12) in the usual continuum approximation for an infinite system

$$
\left\langle\mathcal{H}_{1}\right\rangle=\int d \xi\left(\frac{1}{2}\left(\frac{\left(\partial_{\xi} p\right)^{2}}{1-p^{2}}+\left(1-p^{2}\right)\left(\partial_{\xi} q\right)^{2}\right)-B p\right)
$$

where we have put the magnetic field in $z$ direction, substracted the ground state energy and introduced the canonical conjugate fields $p=\cos \vartheta, q=\varphi ; \xi$ denotes the spatial variable in chain direction. The equations of motion (14) read in continuum approximation:

$$
\begin{aligned}
\partial_{t} q & =-\frac{1}{1-p^{2}} \partial_{\xi}^{2} p-\frac{p}{\left(1-p^{2}\right)^{2}}\left(\partial_{\xi} p\right)^{2}-p\left(\partial_{\xi} q\right)^{2}-B \\
\partial_{t} p & =\left(1-p^{2}\right) \partial_{\xi}^{2} q-2 p\left(\partial_{\xi} p\right) \partial_{\xi} q
\end{aligned}
$$

These equations have well-known soliton solutions (Tjon and Wright 1977):

$$
\begin{aligned}
& p(\xi, t)=1-\frac{2}{(B+\omega) \Gamma^{2}} \operatorname{sech}^{2}\left(\frac{\xi-v t-\xi_{0}}{\Gamma}\right) \\
& q(\xi, t)=q_{0}+\omega t+\frac{v}{2}\left(\xi-v t-\xi_{0}\right)+\tan ^{-1}\left(\frac{2}{v \Gamma} \tanh \left(\frac{\xi-v t-\xi_{0}}{\Gamma}\right)\right)
\end{aligned}
$$

with the soliton width

$$
\Gamma=\frac{1}{\sqrt{B+\omega-\frac{v^{2}}{4}}}>0
$$


and $\xi_{0}, q_{0}$ being constants. The above solution is a pulse soliton with velocity $v$ and an internal frequency $\omega$ constrained by (21). Its energy is calculated from (16):

$$
E=\frac{4}{\Gamma}+\frac{B}{B+\omega} \frac{4}{\Gamma}
$$

The square variance $\left\langle\mathcal{H}_{1}^{2}\right\rangle-\left\langle\mathcal{H}_{1}\right\rangle^{2}=\Omega_{1}+\Omega_{2}$ can be obtained from the continuum version of (15):

$$
\begin{aligned}
& \Omega_{1}=\frac{1}{S}\left(\frac{1}{\Gamma}\left(8(2 B+3 \omega)+\frac{4 \omega^{2}}{B+\omega}\right)-\frac{1}{\Gamma^{3}}\left(16+\frac{8 \omega^{2}}{3(B+\omega)^{2}}+\frac{8 \omega}{B+\omega}\right)\right), \\
& \Omega_{2}=\frac{1}{S^{2}} \frac{4}{3 \Gamma^{3}},
\end{aligned}
$$

where (21) has been used. As explained in the previous section the quantity $\Omega_{1}$ can be interpreted as a natural property of a time-dependent spin state in the semiclassical regime. As a criterion for the importance of quantum effects absent from our ansatz (7) we compare $\Omega_{2}$ with the soliton energy, i. e.

$$
\frac{\sqrt{\Omega_{2}}}{E}=\frac{1}{S \sqrt{\Gamma}} \frac{B+\omega}{2 \sqrt{3}(2 B+\omega)}
$$

We see that higher order quantum corrections to our variational approach are neglegible for solitons with large width and consequently small energy. If this condition is not fullfilled the spin length $S$ becomes significant, in agreement with the work of Balakrishnan and Bishop mentioned in the introduction.

The continuum approximation is a good description of the system if the classical fields $p, q$ vary only weakly on the typical length scale. This means that the soliton width should be significantly larger than the lattice spacing $a=1$, e.g. $\Gamma>10$. Thus, continuum and semiclassical approximation work well in a consistent area of the soliton parameters.

(ii) Next we consider a ferromagnetic Heisenberg chain with a biaxial local anisotropy:

$$
\mathcal{H}_{2}=-J \sum_{n}\left[\hat{\vec{S}}_{n} \hat{\vec{S}}_{n+1}+\tau_{x} \hat{S}_{n}^{x} \hat{S}_{n}^{x}+\tau_{z} \hat{S}_{n}^{z} \hat{S}_{n}^{z}\right]
$$

The square variance of the energy is given by 


$$
\begin{aligned}
&\left\langle\mathcal{H}_{2}^{2}\right\rangle-\left\langle\mathcal{H}_{2}\right\rangle^{2}= \\
&(\hbar S)^{4} J^{2} \sum_{n}\left[\frac { 1 } { 2 S } \left(\vec{s}_{n} \times\left(\vec{s}_{n-1}+\vec{s}_{n+1}\right)\right.\right.\left.+2\left(1-\frac{1}{2 S}\right)\left(\tau_{x}\left(\vec{s}_{n} \times \vec{e}^{x}\right)\left(\vec{s}_{n} \cdot \vec{e}^{x}\right)+\tau_{z}\left(\vec{s}_{n} \times \vec{e}^{z}\right)\left(\vec{s}_{n} \cdot \vec{e}^{z}\right)\right)\right)^{2} \\
&+\frac{1}{8 S^{2}}\left(\left(1-\vec{s}_{n} \vec{s}_{n-1}\right)^{2}+\left(1-\vec{s}_{n} \vec{s}_{n+1}\right)^{2}\right. \\
&+2\left(1-\frac{1}{2 S}\right)\left(\tau_{x}^{2}\left(\vec{s}_{n} \times \vec{e}^{x}\right)^{4}+\tau_{z}^{2}\left(\vec{s}_{n} \times \vec{e}^{z}\right)^{4}\right. \\
&\left.\left.\left.\quad+2 \tau_{x} \tau_{z}\left(\left(\vec{s}_{n} \cdot \vec{e}^{x}\right)\left(\vec{s}_{n} \cdot \vec{e}^{z}\right)-\left(\vec{s}_{n} \cdot\left(\vec{e}^{x} \times \vec{e}^{z}\right)\right)^{2}\right)\right)\right)\right]
\end{aligned}
$$

Proceeding as above one can derive the following solutions of the continuum model (see e. g. Kosevich et al. 1990):

$$
p(\xi, t)=\operatorname{sign}(v) \tanh \left(\frac{\xi-v t+\xi_{0}}{\Gamma}\right) \quad, \quad q(\xi, t)=q_{0}
$$

with

$$
\Gamma=\frac{1}{\sqrt{2\left(1-\frac{1}{2 S}\right)\left(\tau_{z}-\tau_{x} \cos ^{2} q_{0}\right)}} \quad, \quad v^{2}=\frac{\left(\tau_{x} \sin \left(2 q_{0}\right)\right)^{2}\left(1-\frac{1}{2 S}\right)}{2\left(\tau_{z}-\tau_{x} \cos ^{2} q_{0}\right)},
$$

$\xi_{0}, q_{0}$ being constants and $\tau_{z}-\tau_{x} \cos ^{2} q_{0}>0$. The eqs. (28) describe moving kink solitons parametrized by $q_{0}$ with energy $E=\frac{2}{\Gamma}$ above the ground state. For finite $\tau_{x}$ the velocity $v$ vanishes for $q_{0} \in\left\{0, \frac{\pi}{2}\right\}$ and takes its maximum $v_{\max }^{2}=\left|\tau_{x} \cos \left(2 q^{*}\right)\right|$ at $q_{0}=q^{*}$ with

$$
\cos \left(2 q^{*}\right)=2 \frac{\tau_{z}}{\tau_{x}}-1-\operatorname{sign}\left(\frac{\tau_{z}}{\tau_{x}}\right) \sqrt{\left(2 \frac{\tau_{z}}{\tau_{x}}-1\right)^{2}-1}
$$

Inserting eqs. (28) into the continuum version of (27) leads to a divergence in $\Omega_{2}$ arising from the term proportional to $\tau_{x}^{2}$. This contribution is almost uniform over the whole system except for an area around the center of the kink. Moreover, the same divergence occurs if we evaluate $\Omega_{2}$ for the classical ground state of the model (26). The explanation for this behaviour is that, different from the previous model, the quantum mechanical ground state of (26) is not described exactly by our ansatz (7). Thus, the divergent contribution in order $\frac{1}{S^{2}}$ is clearly an artifact of our variational approach due to a more complicated structure of the quantum mechanical ground state, which does not correspond trivially to the classical ground state solution. Note that a similar infinite term in $\Omega_{2}$ arises for the classical ground state of an antiferromagnetic Heisenberg model, e. g. (12) with $J<0$. Here the divergence in order $\frac{1}{S^{2}}$ is due to the contribution from the isotropic exchange in the Hamiltonian.

Since we are interested in physically valid quantum fluctuations in time-dependent excitations within a semiclassical approach, we renormalize $\left\langle\mathcal{H}_{2}^{2}\right\rangle-\left\langle\mathcal{H}_{2}\right\rangle^{2}$ by neglecting $\Omega_{2}$. The remaining contribution is calculated easily giving

$$
\Omega_{1}=\frac{1}{S} \frac{v^{2}}{\Gamma}=\frac{1}{S} \frac{\left(\tau_{x} \sin \left(2 q_{0}\right)\right)^{2}\left(1-\frac{1}{2 S}\right)^{\frac{3}{2}}}{\left(2\left(\tau_{z}-\tau_{x} \cos ^{2} q_{0}\right)\right)^{\frac{1}{2}}} .
$$


This leading order of energy variance vanishes of course in the static case $q_{0} \in\left\{0, \frac{\pi}{2}\right\}$ and takes its maximum at $q_{0}=q^{* *}$ where

$$
\cos \left(2 q^{* *}\right)=2\left(2 \frac{\tau_{z}}{\tau_{x}}-1\right)-2 \operatorname{sign}\left(\frac{\tau_{z}}{\tau_{x}}\right) \sqrt{\left(2 \frac{\tau_{z}}{\tau_{x}}-1\right)^{2}-\frac{3}{4}}
$$

Obviously, $q^{*}$ and $q^{* *}$ do not coincide.

In the case $\tau_{x}=0$ the kink solitons become static, and the exact quantum ground state is again the trivial ferromagnetic state. Thus, $\Omega_{1}=0$ and in $\Omega_{2}$ no unphysical divergency arises:

$$
\Omega_{2}=\frac{1}{S^{2}} \frac{1}{3 \sqrt{2}} \sqrt{\left(1-\frac{1}{2 S}\right)} \tau_{z}^{\frac{3}{2}}\left(1+4\left(1-\frac{1}{2 S}\right)\right)
$$

Comparing $\sqrt{\Omega_{2}}$ with the energy of the kink excitation, we again conclude that the semiclassical description should be valid for solitons with large width and low energy.

$\underline{\text { (iii) }}$ The isotropic Heisenberg ferromagnet on a two-dimensional infinite square lattice is given by

$$
\mathcal{H}_{3}=-J \sum_{m, n} \hat{\vec{S}}_{m, n}\left(\hat{\vec{S}}_{m+1, n}+\hat{\vec{S}}_{m, n+1}\right)
$$

In appropriate units we find in the continuum approximation

$$
\left\langle\mathcal{H}_{3}\right\rangle=\frac{1}{2} \int d^{2} x\left(\frac{(\nabla p)^{2}}{1-p^{2}}+\left(1-p^{2}\right)(\nabla q)^{2}\right)
$$

Eq. (35) defines a two-dimensional conformal invariant field theory that has been investigated by Belavin and Polyakov (1975). The fields $p, q$ parametrize points on the unit sphere $S^{2}$. Compactifying the two-dimensional plane by an infinite point we can consider the solutions of the Hamiltonian (35) as mappings $S^{2} \rightarrow S^{2}$. These mappings can be classified in homotopical classes characterized by the degree of mapping $d$ with

$$
|d|=\frac{1}{8 \pi} \int d^{2} x \varepsilon_{\alpha \beta \gamma} \varepsilon_{\mu \nu} s^{\alpha} \frac{\partial s^{\beta}}{\partial x_{\mu}} \frac{\partial s^{\gamma}}{\partial x_{\nu}}=\frac{1}{4 \pi} \int d \varphi(\vec{x}) d(\cos \vartheta(\vec{x})),
$$

which is the number of times that the sphere is covered in the course of mapping. As shown by the above authors it holds

$$
\left\langle\mathcal{H}_{3}\right\rangle=4 \int d^{2} z \frac{1}{\left(1+|w|^{2}\right)^{2}}\left(\frac{\partial w}{\partial z} \frac{\partial \bar{w}}{\partial \bar{z}}+\frac{\partial \bar{w}}{\partial z} \frac{\partial w}{\partial \bar{z}}\right) \geq 4 \pi|d|
$$

with $w(z, \bar{z})=\cot \frac{\vartheta}{2} e^{\imath \varphi}$ and $z=x_{1}+\imath x_{2}$. The minimum in (37) is realized by instanton solutions that are given by arbitrary meromorphic functions $w(z)$ :

$$
w(z)=\prod_{i}\left(\frac{z-a_{i}}{R}\right)^{m_{i}} \cdot \prod_{i}\left(\frac{R}{z-b_{i}}\right)^{n_{i}}
$$

with $m_{i}, n_{i}>0$ and $a_{i} \neq b_{j}$ for all $i, j$ and the degree given by $d=\max \left\{\sum_{i} m_{i}, \sum_{i} n_{i}\right\}$. The real scale parameter $R$ does not influence the energy of these static excitations due to the 
conformal invariance of the model.

Let us now consider a single instanton simply given by $w=\left(\frac{z}{R}\right)^{\nu}, d=|\nu|$, or

$$
p=\frac{|z|^{2 \nu}-R^{2 \nu}}{|z|^{2 \nu}+R^{2 \nu}} \quad, \quad q=\nu \arg (z)
$$

Clearly we have $\Omega_{1}=0$, and for $\Omega_{2}$ one obtains

$$
\Omega_{2}=\frac{1}{S^{2} R^{2}} \frac{2 \pi^{2}}{3} \frac{\nu^{2}-1}{\sin \frac{\pi}{|\nu|}} \quad, \quad|\nu|>1
$$

and $\Omega_{2}=\frac{1}{S^{2} R^{2}} \frac{4 \pi}{3}$ for $|\nu|=1$. In contrast to the energy of the continuum model this quantity is not independent of $R$, but scales with $\frac{1}{R^{2}}$. Again, for the semiclassical description to be valid, $\sqrt{\Omega_{2}}$ should be small compared with the energy $\left\langle\mathcal{H}_{3}\right\rangle=4 \pi d$. This can be achieved for arbitrarily high energies provided that the width of the instanton is sufficiently large.

Next we consider a solution consisting of two instantons separated by distance $2 l$,

$$
w(z)=\left(\frac{z-l}{R}\right)^{\mu}\left(\frac{z+l}{R}\right)^{\nu},
$$

and find

$$
\Omega_{2}=\frac{1}{2 S^{2}} \int d^{2} z\left(\frac{\left|\frac{\partial w}{\partial z}\right|^{2}}{\left(1+|w|^{2}\right)^{2}}\right)^{2}=\frac{1}{2 S^{2} R^{2}} F\left(\mu, \nu ; \frac{l}{R}\right)
$$

with

$$
F(\mu, \nu ; a)=\int d^{2} z\left(\frac{|z-a|^{\mu-1}|z+a|^{\nu-1}((\mu+\nu) z+(\mu-\nu) a)}{1+|z-a|^{2 \mu}|z+a|^{2 \nu}}\right)^{4}
$$

Again, the square variance of the energy scales with $\frac{1}{R^{2}}$. Unfortunately, the quantity $F(\mu, \nu ; a)$ cannot be expressed by elementary functions; e.g. in the case $\mu=\nu$ one obtains after some algebra:

$$
F(\mu, \mu ; a)=a^{-8 \mu-2} \int d^{2} x \frac{|\vec{x}|^{4 \mu-4}|\vec{x}+\vec{e}|}{\left(a^{-4 \mu}+|\vec{x}|^{2 \mu}\right)^{4}}
$$

with $\vec{e}$ being an arbitrary unit vector.

(iv) Finally we consider an anisotropic two-dimensional Heisenberg Hamiltonian:

$$
\mathcal{H}_{4}=-J \sum_{m, n}\left[\hat{\vec{S}}_{m, n}\left(\hat{\vec{S}}_{m+1, n}+\hat{\vec{S}}_{m, n+1}\right)-\lambda \hat{S}_{m, n}^{z} \hat{S}_{m, n}^{z}\right]
$$

Choosing $\lambda>0$ and the lattice lying in the $x$-y-plane the classical ground state is given by a parallel spin configuration in this easy plane. Further we have:

$$
\begin{aligned}
&\left\langle\mathcal{H}_{4}^{2}\right\rangle-\left\langle\mathcal{H}_{4}\right\rangle^{2}= \\
&(\hbar S)^{4} J^{2} \sum_{m, n}\left[\frac { 1 } { 2 S } \left(\vec{s}_{m, n} \times\left(\vec{s}_{m-1, n}+\vec{s}_{m+1, n}+\vec{s}_{m, n-1}+\vec{s}_{m, n+1}\right)\right.\right. \\
&\left.\quad-2\left(1-\frac{1}{2 S}\right) \lambda\left(\vec{s}_{m, n} \cdot \vec{e}^{z}\right)\left(\vec{s}_{m, n} \times \vec{e}^{z}\right)\right)^{2}
\end{aligned}
$$




$$
\begin{gathered}
+\frac{1}{8 S^{2}}\left(\left(1-\vec{s}_{m, n} \vec{s}_{m-1, n}\right)^{2}+\left(1-\vec{s}_{m, n} \vec{s}_{m+1, n}\right)^{2}+\left(1-\vec{s}_{m, n} \vec{s}_{m, n-1}\right)^{2}+\left(1-\vec{s}_{m, n} \vec{s}_{m, n+1}\right)^{2}\right. \\
\left.\left.+2\left(1-\frac{1}{2 S}\right) \lambda^{2}\left(\vec{s}_{m, n} \times \vec{e}^{z}\right)^{4}\right)\right]
\end{gathered}
$$

For sufficiently large $\lambda$ planar vortices are stable excitations (Gouvea et al. 1989). These objects are given in a continuum approximation by

$$
p(\vec{x}, t)=0 \quad, \quad q(\vec{x}, t)=\nu \tan ^{-1}\left(\frac{x_{2}}{x_{1}}\right)
$$

with energy $E=\pi \nu^{2} \ln \left(\frac{L}{a}\right)$, where we have chosen appropriate units as before. The integer $\nu$ is called the vorticity, $L$ is the size of the system and $a=1$ is the lattice spacing, which is a lower cutoff for the integration. The energy diverges logarithmically with the system size, due to the fact that the spin configuration is not parallel far away from the vortex center. In this sense, the planar vortex is not localized.

Inserting such a static planar solution into (46) obviously gives $\Omega_{1}=0$ and in $\Omega_{2}$ a contribution proportional to $\lambda^{2}$ that diverges quadratically with growing $L$. This effect is completely analogous to the divergence in the model (26) and has its reason in an unexact description of the quantum ground state within our variational ansatz. So we renormalize $\Omega_{2}$ by neglecting this artificial term, which is identical for any planar solution and therefore in particular not sensitive for the vortex (47). The remaining expression for the quantum mechanical variance of energy is:

$$
\Delta E=\frac{1}{2 S} \sqrt{\frac{\pi}{6}} \nu^{2} \sqrt{1-\frac{1}{L^{2}}}
$$

In a classical easy-plane model of the above type a topological phase transition, or KosterlitzThouless-transition, occurs at a certain temperature $T_{K T}$ (Kosterlitz and Thouless 1973, Kosterlitz 1974). Below this temperature vortices and antivortices (differing by the sign of the topological charge $\nu$ ) are bound in pairs, while they become mobile above $T_{K T}$.

Now we use our result (48) to estimate quantum corrections to the value of the critical temperature $T_{K T}$. We assume that only vortices with $|\nu|=1$ are present in our system and follow some rough approximations in the given references.

In the free energy $F=E-T S$ the quantum fluctuations will give an additional contribution to the entropy $S$. For a single vortex the classical entropy is $S=\ln \left(L^{2}\right)$, since the vortex can be placed anywhere in the system. In our semiclassical description we have to take into account the uncertaincy of the energy:

$$
S=\ln \left(L^{2}\left(1+\frac{\Delta E}{E}\right)\right)=2 \ln L+\frac{\Delta E}{E}+O\left(\frac{1}{S^{2}}\right)
$$

The entropy term will dominate in the free energy above a temperature

$$
T=\frac{\pi}{2} \frac{1}{1+\frac{\Delta E}{2 \pi(\ln L)^{2}}}=\frac{\pi}{2}\left(1-\frac{\Delta E}{2 \pi(\ln L)^{2}}\right)+O\left(\frac{1}{S^{2}}\right),
$$

which reduces to the classical estimate $T_{K T}=\frac{\pi}{2}$ for $L \rightarrow \infty$. Thus, we conclude that in an infinite system there is no quantum correction to the Kosterlitz-Thouless-temperature in first order in $\frac{1}{S}$, but a logarithmic finite-size contribution arises in this order. 


\section{Conclusions}

In this work we investigate a large family of spin models in the semiclassical regime with respect to quantum fluctuations. Our main result is given by eqs. (10), (11) which provide a physically relevant expression of quantum fluctuations of the energy in low orders in $\frac{1}{S}$. In particular, the contribution in first order is purely due to the time-dependence of the spin configuration in semiclssical description. For a static spin configuration the square variance of the energy is of order $\frac{1}{S^{2}}$. The term of first order is a natural property of a state vector with a non-trivial time- dependence, which is consequently not an eigenstate of the Hamiltonian. The terms of higher order can often be used as criterion of the validity of the semiclassical approach.

These findings are valid for a large variety of spin models in arbitrary dimension. In the previous section we have illustrated this by several prominent spin models.

Acknowledgement: The authors are grateful to Holger Frahm for useful discussions. 


\section{A Expectation values within coherent states}

Let $\mathcal{P}\left[\hat{S^{+}}, \hat{S^{-}}, \hat{S^{z}}\right]$ be an arbitrary product of operator-valued spin components. With $U$ given in eq. (3) we have

$$
\left\langle S ; \vartheta, \varphi\left|\mathcal{P}\left[\hat{S}^{+}, \hat{S}^{-}, \hat{S}^{z}\right]\right| S ; \vartheta, \varphi\right\rangle=\left\langle S\left|\mathcal{P}\left[U^{+} \hat{S}^{+} U, U^{+} \hat{S}^{-} U, U^{+} \hat{S}^{z} U\right]\right| S\right\rangle
$$

It holds:

$$
\begin{array}{r}
U^{+} \hat{S}^{+} U=e^{\imath \varphi}\left(\cos ^{2}\left(\frac{\vartheta}{2}\right) \hat{S}^{+}-\sin ^{2}\left(\frac{\vartheta}{2}\right) \hat{S}^{-}+\sin \vartheta \hat{S}^{z}\right) \\
U^{+} \hat{S}^{z} U=\left(-\frac{1}{2} \sin \vartheta \hat{S}^{+}-\frac{1}{2} \sin \vartheta \hat{S}^{-}+\cos \vartheta \hat{S}^{z}\right) .
\end{array}
$$

Applying $\hat{S}^{+}, \hat{S}^{-}$on states typed $|S-m\rangle$ gives a contribution of leading order $\sqrt{S}$, while $\hat{S}^{z}$ gives a factor of leading order $S$. By such arguments it is easily seen that the leading term in eq. (51) is proportional to the product of the classical spin components given in $\vec{s}_{\vartheta, \varphi}$ :

$$
\lim _{\substack{S \rightarrow \infty \\ \hbar S=S^{c l}=\text { const. }}}\left\langle S ; \vartheta, \varphi\left|\mathcal{P}\left[\hat{S}^{+}, \hat{S}^{-}, \hat{S}^{z}\right]\right| S ; \vartheta, \varphi\right\rangle=\mathcal{P}\left[S^{c l} s_{\vartheta, \varphi}^{+}, S^{c l} s_{\vartheta, \varphi}^{-}, S^{c l} s_{\vartheta, \varphi}^{z}\right],
$$

where $(\hbar S)=S^{c l}=$ const. is the classical spin length. In the classical limit taken above all terms of higher order in $\frac{1}{S}$ (or equivalently in $\hbar$ ) drop. Of course, the details of these quantum contributions depend on the structure of $\mathcal{P}\left[\hat{S}^{+}, \hat{S}^{-}, \hat{S}^{z}\right]$, i.e. the ordering of the spin operators. Using eqs. (52), (53) expectation values of type (51) can be calculated easily. Here we give a list of diagonal elements useful for the derivation of eq. (10):

$$
\begin{gathered}
\left\langle S ; \vartheta, \varphi\left|\hat{S}^{z} \hat{S}^{z}\right| S ; \vartheta, \varphi\right\rangle=(\hbar S)^{2}\left(\cos ^{2} \vartheta+\frac{1}{2 S} \sin ^{2} \vartheta\right) \\
\left\langle S ; \vartheta, \varphi\left|\hat{S}^{+} \hat{S}^{-}+\hat{S}^{-} \hat{S}^{+}\right| S ; \vartheta, \varphi\right\rangle=2(\hbar S)^{2}\left(\sin ^{2} \vartheta+\frac{1}{S}\left(1+\cos ^{2} \vartheta\right)\right) \\
\left\langle S ; \vartheta, \varphi\left|\hat{S}^{+} \hat{S}^{z}+\hat{S}^{z} \hat{S}^{+}\right| S ; \vartheta, \varphi\right\rangle=2(\hbar S)^{2}\left(1-\frac{1}{2 S}\right) e^{\imath \varphi} \cos \vartheta \sin \vartheta \\
\left\langle S ; \vartheta, \varphi\left|\hat{S}^{z} \hat{S}^{z} \hat{S}^{z}\right| S ; \vartheta, \varphi\right\rangle=(\hbar S)^{3}\left(\cos ^{3} \vartheta+\left(\frac{3}{2 S}-\frac{1}{2 S^{2}}\right) \cos \vartheta \sin ^{2} \vartheta\right) \\
\left\langle S ; \vartheta, \varphi\left|\hat{S}^{+} \hat{S}^{z} \hat{S}^{+}\right| S ; \vartheta, \varphi\right\rangle=(\hbar S)^{3}\left(1-\frac{1}{2 S}\right)\left(1-\frac{1}{S}\right) e^{\imath 2 \varphi} \cos \vartheta \sin ^{2} \vartheta \\
\left\langle S ; \vartheta, \varphi\left|\hat{S}^{z} \hat{S}^{+} \hat{S}^{z}\right| S ; \vartheta, \varphi\right\rangle=(\hbar S)^{3}\left(1-\frac{1}{S}\right) e^{\imath \varphi}\left(\cos ^{2} \vartheta \sin \vartheta+\frac{1}{2 S} \sin ^{3} \vartheta\right) \\
\left\langle S ; \vartheta, \varphi\left|\hat{S}^{+} \hat{S}^{z} \hat{S}^{z}+\hat{S}^{z} \hat{S}^{z} \hat{S}^{+}\right| S ; \vartheta, \varphi\right\rangle \\
=2(\hbar S)^{3} e^{\imath \varphi}\left(\left(1-\frac{1}{2 S}\right)\left(1-\frac{1}{S}\right) \cos ^{2} \vartheta \sin \vartheta+\frac{1}{2 S} \sin \vartheta\right) \\
\left\langle S ; \vartheta, \varphi\left|\hat{S}^{z} \hat{S}^{z} \hat{S}^{z} \hat{S}^{z}\right| S ; \vartheta, \varphi\right\rangle \\
=(\hbar S)^{4}\left(\cos ^{4} \vartheta+\left(\frac{3}{S}-\frac{5}{2 S^{2}}+\frac{5}{8 S^{3}}\right) \cos ^{2} \vartheta \sin ^{2} \vartheta+\left(\frac{1}{2 S^{2}}+\frac{1}{8 S^{3}}\right) \sin ^{2} \vartheta\right)
\end{gathered}
$$




$$
\begin{aligned}
& \left\langle S ; \vartheta, \varphi\left|\hat{S}^{z} \hat{S}^{z}\left(\hat{S}^{+} \hat{S}^{-}+\hat{S}^{-} \hat{S}^{+}\right)+\left(\hat{S}^{+} \hat{S}^{-}+\hat{S}^{-} \hat{S}^{+}\right) \hat{S}^{z} \hat{S}^{z}\right| S ; \vartheta, \varphi\right\rangle \\
& =2(\hbar S)^{4}\left(\left(2-\frac{6}{S}+\frac{5}{S^{2}}-\frac{5}{4 S^{3}}\right) \cos ^{2} \vartheta \sin ^{2} \vartheta+\frac{2}{S} \cos ^{2} \vartheta+\left(\frac{1}{S}+\frac{1}{4 S^{3}}\right) \sin ^{2} \vartheta\right) \\
& \left\langle S ; \vartheta, \varphi\left|\hat{S}^{z} \hat{S}^{z}\left(\hat{S}^{+} \hat{S}^{+}+\hat{S}^{-} \hat{S}^{-}\right)+\left(\hat{S}^{+} \hat{S}^{+}+\hat{S}^{-} \hat{S}^{-}\right) \hat{S}^{z} \hat{S}^{z}\right| S ; \vartheta, \varphi\right\rangle \\
& =2(\hbar S)^{4} \cos (2 \varphi)\left(\left(2-\frac{6}{S}+\frac{5}{S^{2}}-\frac{5}{4 S^{3}}\right) \cos ^{2} \vartheta \sin ^{2} \vartheta+\left(\frac{1}{S}-\frac{1}{4 S^{3}}\right) \sin ^{2} \vartheta\right)
\end{aligned}
$$

\section{B Coherent states and the classical limit of Spin systems}

Let us consider a system of spins $\left\{\vec{S}_{i}\right\}_{i \in I}$. The dynamics are given by a quantum Hamiltonian $\mathcal{H}\left[\left\{\hat{\vec{S}}_{i}\right\}_{i \in I}\right]$, which is taken to be an arbitrary polynomial in the spin components.

We now evaluate the Heisenberg equation of motion for the $i$-th spin at a chosen time $t=t_{0}$ in terms of a tensor product of spin-coherent states

$$
\left|\psi\left(t_{0}\right)\right\rangle=\bigotimes_{i \in I}\left|S_{i} ; \vartheta_{i}, \varphi_{i}\right\rangle
$$

and denote by $\langle\cdot\rangle$ an expectation value in the state (65):

$$
\left(\frac{d}{d t}\left\langle\hat{\vec{S}}_{i}\right\rangle\right)_{t=t_{0}}=\frac{1}{\imath \hbar}\left(\left\langle\left[\hat{\vec{S}}_{i}, \mathcal{H}\right]\right\rangle\right)_{t=t_{0}}
$$

The right hand side of this equation can be evaluated straightforewardly in terms of the variables $\vartheta_{i}, \varphi_{i}$, while for the left hand side we need information about the time evolution of the wave function $|\psi(t)\rangle$.

The time evolution of the spin expectation values is

$$
\begin{array}{r}
\left\langle\psi(t)\left|\hat{\vec{S}}_{i}\right| \psi(t)\right\rangle=\left\langle\psi\left(t_{0}\right)\left|\exp \left(+\frac{\imath}{\hbar} \mathcal{H}\left(t-t_{0}\right)\right) \hat{\vec{S}}_{i} \exp \left(-\frac{\imath}{\hbar} \mathcal{H}\left(t-t_{0}\right)\right)\right| \psi\left(t_{0}\right)\right\rangle \\
=\left\langle\hat{\vec{S}}_{i}\right\rangle_{t_{0}}+\frac{\imath\left(t-t_{0}\right)}{\hbar}\left\langle\left[\mathcal{H}, \hat{\vec{S}}_{i}\right]\right\rangle_{t_{0}}+\frac{1}{2}\left(\frac{\imath\left(t-t_{0}\right)}{\hbar}\right)^{2}\left\langle\left[\mathcal{H},\left[\mathcal{H}, \hat{\vec{S}}_{i}\right]\right]\right\rangle_{t_{0}}+\cdots
\end{array}
$$

The diagonal elements in the expansion (67) are products of expressions of the form (51) and therefore reduce in the limit $S \rightarrow \infty, \hbar \rightarrow 0, \hbar S=$ const to the classical values. E.g. for the first commutator we have

$$
\lim _{\substack{S_{j} \rightarrow \infty \\ \hbar S_{j}=S_{j}^{c l}=\text { const. } \\ j \in I}} \frac{\imath}{\hbar}\left\langle\left[\mathcal{H}, \hat{\vec{S}}_{i}\right]\right\rangle_{t_{0}}=-S_{i}^{c l} \vec{s}_{i} \times \frac{\partial \mathcal{H}\left[\left\{S_{j}^{c l} \vec{s}_{j}\right\}_{j \in I}\right]}{\partial\left(S_{i}^{c l} \vec{s}_{i}\right)}
$$

with $\vec{s}_{i}=\left(\sin \vartheta_{i} \cos \varphi_{i}, \sin \vartheta_{i} \sin \varphi_{i}, \cos \vartheta_{i}\right)$. The right hand side of this equation is nothing but the classical Poisson bracket $\left\{S_{i}^{c l} \vec{s}_{i}, \mathcal{H}\right\}$ occuring in the well-known Landau-Lifshitz-equation, i.e. the equation of motion for a classical spin system. Similar arguments hold for the higher commutators in (67), and we end up with

$$
\lim _{\substack{S_{j} \rightarrow \infty \\ S_{j}=S_{j}^{c l}=\text { const. } \\ j \in I}}\left\langle\psi(t)\left|\hat{\vec{S}}_{i}\right| \psi(t)\right\rangle=S_{i}^{c l} \vec{s}_{i}+\left(t-t_{0}\right)\left\{S_{i}^{c l} \vec{s}_{i}, \mathcal{H}\right\}+\frac{\left(t-t_{0}\right)^{2}}{2}\left\{\left\{S_{i}^{c l} \vec{s}_{i}, \mathcal{H}\right\}, \mathcal{H}\right\}+\cdots
$$


Thus, we have confirmed that the classical limit of the quantum mechanical time evolution (67) reproduces the motion of a classical spin vector in the case of an arbitrary Hamiltonian. By similar considerations one can convince oneself that minimizing the action

$$
\mathcal{S}=\int_{t_{1}}^{t_{2}} d t\left\langle\psi(t)\left|\imath \hbar \frac{d}{d t}-\mathcal{H}\right| \psi(t)\right\rangle
$$

in the classical limit with respect to the functions $\vartheta_{i}(t), \varphi_{i}(t)$ also leads to the classical equations. This should be expected since the variation principle concerning (70) (with arbitrary wave function $|\psi(t)\rangle$ ) is equivalent to the Heisenberg equation of motion.

\section{References}

L. G. de Azevedo, M. A. Moura, C. C. Cordeiro, B. Zeks 1982, J. Phys. C: Solid State Phys. 15, 7391

R. Balakrishnan, A. R. Bishop 1985, Phys. Rev. Lett. 55, 537

R. Balakrishnan, A. R. Bishop 1989, Phys. Rev. B 40, 9194

R. Balakrishnan, J. A. Holyst, A. R. Bishop 1990, J. Phys: Condens. Matter 2, 1869

A. A. Belavin, A. M. Polyakov 1975, JETP Lett. 22, 245

H. Bethe 1931, Z. Phys. 71, 205

R. Ferrer, J. Pozo 1988, Physica B 153, 225

H. C. Fogedby 1980, J. Phys A: Math. Gen. 13, 1467

H. Frahm, J. A. Holyst 1989, J. Phys: Condens. Matter 1, 3083

M. E. Gouvea, G. M. Wysin, A. R. Bishop, F. G. Mertens 1989, Phys. Rev. B 39, 11840

F. D. M. Haldane 1986, Phys. Rev. Lett 57, 1488

D. V. Kapor, M. J. Skrinjar, S. D. Stojanovic 1990 in Lecture Notes in Physics, Vol. 353, edited by M. Barthes and J. Leon, Springer

A. M. Kosevich, B. A. Ivanov, A. S. Kovalev 1990, Phys. Rep. 194, 117

J. M. Kosterlitz, D. J. Thouless 1973, J. Phys. C: Solid State Phys. 6, 1181

J. M. Kosterlitz 1974, J. Phys. C: Solid State Phys. 7, 1046

J. M. Radcliffe 1971, J. Phys. A: Gen. Phys. 4, 313

E. K. Sklyanin 1979, LOMI preprint

L. A. Takhtajan 1977, Phys. Lett. 64A, 235,

J. Tjon, J. Wright 1977, Phys. Rev. B 15, 3470 